\title{
DETERMINANTES DA DEMANDA DE GASOLINA C NO ESTADO DE MINAS GERAIS, 2002 A $2010^{1}$
}

\author{
Rosangela Aparecida Soares Fernandes ${ }^{2}$ \\ Cristiane Marcia dos Santos ${ }^{3}$
}

Sarah Lorena Peixoto ${ }^{4}$

Resumo: Este trabalho teve como objetivo analisar a demanda de gasolina $\mathrm{C}$ no Estado de Minas Gerais, de janeiro de 2002 a dezembro 2010. Os resultados sugeriram que a demanda de gasolina $\mathrm{C}$ é influenciada pelo seu preço, pelo preço do álcool hidratado e pela renda do consumidor. Verificou-se que a demanda foi inelástica em relação ao preço da gasolina $\mathrm{C}$ no curto e no longo prazo e elástica em relação à renda no longo prazo, enquanto no curto prazo ela se revelou inelástica em relação à renda. $\mathrm{O}$ álcool hidratado é um substituto imperfeito da gasolina $\mathrm{C}$, tanto no curto quanto no longo prazo.

Palavras-chave: demanda, gasolina C, Minas Gerais, elasticidade.

Abstract: This study aimed to analyze the demand for gasoline $\mathrm{C}$ in Minas Gerais, from January 2002 to December 2010. The results suggested that gasoline demand is influenced by the $\mathrm{C}$ of $\mathrm{C}$ gasoline prices and ethanol and the consumer's income. It was found that demand was inelastic to the price of gasoline $\mathrm{C}$, in the short and long term, and elastic in relation to incomes in the long term, while in the short term proved to be inelastic to income. Hydrated alcohol is an imperfect substitute for gasoline $\mathrm{C}$, both the short and long term.

Keywords: demand, gasoline C, Minas Gerais, elasticity.

1 Recebido em: 13/06/2012. Aceito em: 27/08/2012.

2 Doutora em Economia Aplicada pela Universidade Federal de Viçosa. Professora Adjunta pela Universidade Federal de Ouro Preto. E-mail: roaeconomista@yahoo.com.br

3 Doutora em Economia Aplicada pela Universidade Federal de Viçosa. Professora Adjunta pela Universidade Federal de Ouro Preto. E-mail: cristiane@icsa.ufop.br

4 Graduanda em Engenharia de Produção pela Universidade Federal de Ouro Preto. E-mail:sarahpeixoto@ yahoo.com.br 


\section{Introdução}

De acordo com Souza (2010), a gasolina C e o álcool hidratado são os principais combustíveis para o abastecimento de veículos leves no Brasil, e a investigação a respeito de suas demandas evidencia relevantes indicadores sobre o comportamento do mercado consumidor. Existem trabalhos de vários pesquisadores no mundo que buscaram estimar as elasticidades-preço e renda da demanda por gasolina $\mathrm{C}$, e em menor número, as elasticidades da demanda por álcool hidratado para diferentes países. No Brasil, a demanda por gasolina C também tem sido frequentemente analisada, como, por exemplo, nos trabalhos mais recentes de Nappo (2007), Schünemann (2007), Vilela e Júnior (2010) e Souza (2010).

Este artigo teve como objetivo analisar a demanda de gasolina $\mathrm{C}$ no Estado de Minas Gerais, no período de janeiro de 2002 a dezembro de 2010, com base no Modelo de Correção de Erro (MCE). Ao que tudo indica, ainda são escassos estudos que analisam a demanda de gasolina C nesse Estado para o período mais recente. Diante disso, espera-se que a análise feita neste artigo permita obter uma melhor compreensão a respeito dos determinantes da demanda de gasolina $\mathrm{C}$ em Minas Gerais, bem como o comportamento do consumidor no que se refere às variações no preço desse combustível, do álcool hidratado e da renda.

De acordo com os dados da Agência Nacional do Petróleo, Gás Natural e Biocombustíveis - ANP (2011), as vendas de gasolina C no Brasil têm apresentando expressivo crescimento no período recente. De 2002 a 2010, o volume vendido desse combustível pelas distribuidoras aumentou cerca de $24 \%$, passando de 22.610.257 para 29.843.665m³ . No Estado de Minas Gerais, a expansão das vendas de gasolina $\mathrm{C}$ vem seguindo essa mesma tendência, entretanto o crescimento no período 2002-2010 se revelou ainda mais significativo quando comparado ao verificado no mercado nacional, em torno de 36,6\%. Apesar dessa expansão da demanda de gasolina $\mathrm{C}$ em Minas Gerais, pouco se conhece a respeito de seus determinantes. O Estado é o segundo maior mercado consumidor 
de gasolina C da região Sudeste, ficando atrás somente de São Paulo. Mediante esse cenário, justifica-se a escolha desse mercado relevante geográfico para a realização do presente trabalho.

$\mathrm{O}$ artigo está dividido em quatro seções, além dessa introdução. $\mathrm{Na}$ segunda seção, foi feita uma revisão da literatura recente sobre a demanda dos combustíveis líquidos no Brasil. Na terceira, foi apresentado o modelo analítico, com base na especificação do modelo econométrico e no método de estimação, tendo sido definidos as variáveis e os dados utilizados. Na quarta, foram analisados e discutidos os resultados das estimativas do modelo. Na quinta, foi apresentada uma síntese conclusiva desse artigo.

\section{Estudos recentes sobre a demanda de combustíveis no Brasil}

O estudo da demanda por combustíveis automotivos é tema em diversos trabalhos acadêmicos em todo o mundo. Especificamente, no Brasil, existem várias pesquisas que se baseiam em diferentes métodos empíricos para estimar a demanda por combustíveis e suas elasticidades. Nesta seção, foi feita uma breve revisão da literatura recente sobre a demanda dos combustíveis líquidos, gasolina $\mathrm{C}$ e álcool hidratado, com aplicações para o mercado brasileiro.

Burnquist e Bacchi (2001) estimaram as elasticidades de curto e longo prazo da demanda por gasolina no Brasil para o período de 1973 a 1998, utilizando um Modelo de Correção de Erros. Os resultados encontrados neste trabalho sugeriram que a demanda por gasolina $\mathrm{C}$ era inelástica em relação ao preço no curto prazo e no longo prazo. Entretanto, para ambos os períodos, as estimativas foram menores do que a unidade. Além disso, os autores concluíram que um modelo, baseado apenas na renda e no preço do bem, apresentava estimativas da demanda desse combustível robustas e confiáveis. 
Alves e Bueno (2003) também estimaram as elasticidades de curto e longo prazo da demanda por gasolina no Brasil, utilizando das técnicas de cointegração no período de 1973 a 1999. Neste trabalho, adicionou-se o preço do álcool hidratado à demanda de gasolina a fim de se verificar a possibilidade de substituição entre ambos os combustíveis líquidos nesse período. Os resultados mostraram que o álcool pode ser considerado um substituto imperfeito da gasolina $\mathrm{C}$, no curto e no longo prazo, uma vez que a elasticidade cruzada foi estatisticamente significativa a 15\%, nível que os autores consideraram razoável em razão do tamanho limitado da amostra de dados disponíveis.

Roppa (2005) estimou as elasticidades-preço direta, cruzada e renda da demanda de gasolina C no Brasil, para o período de 1973 a 2003, utilizando a técnica de cointegração. A especificação de seu modelo é semelhante àquela realizada no trabalho de Alves \& Bueno (2003). O objetivo principal deste trabalho foi investigar as possibilidades de o álcool hidratado se consolidar como um combustível substituto da gasolina no mercado brasileiro para o período de análise. Os resultados obtidos sugeriram que o álcool era um substituto imperfeito para a gasolina, ao menos no longo prazo. No curto prazo, os resultados dos testes econométricos para o preço do álcool não se revelaram estatisticamente significativos. Conclui-se que os consumidores não são muito sensíveis às modificações no preço da gasolina. A elasticidaderenda da demanda de gasolina evidenciou que os consumidores estavam mais dispostos a despender sua renda com gasolina no curto prazo do que no longo prazo.

O estudo de Bacchi (2005) teve como objetivo a construção e a estimação de um modelo de Autorregressão Vetorial (VAR) que explicasse o comportamento dos preços dos produtos no setor sucroalcooleiro brasileiro, considerando a inter-relação existente com o setor de combustíveis fósseis, durante o período de julho de 2001 a agosto de 2004. Os resultados evidenciaram que as variações no preço da gasolina $\mathrm{C}$ ao consumidor tiveram efeito imediato e de grande magnitude sobre o preço do álcool hidratado, indicando o elevado grau de substituição 
entre os produtos mencionados. Além disso, o etanol hidratado revelouse um bem inferior, pelo fato de ser grande o uso deste produto como substituto da gasolina $\mathrm{C}$.

Silvério (2007) avaliou o impacto que a introdução dos veículos flex teve sobre as elasticidades preço e renda da demanda por álcool com base nos dados de vendas do álcool e dos preços ao consumidor no Estado do Rio de Janeiro. Para tal, utilizou-se da técnica de cointegração para a estimação das elasticidades e de uma variável binária de inclinação associada à introdução dos veículos flex. Os resultados encontrados sugeriram que a demanda era elástica $(8,623)$ em relação à renda, inelástica em relação ao preço e o coeficiente da variável binária de inclinação foi de $-0,295$.

Nappo (2007) analisou o impacto das vendas dos veículos flex-fuel sobre a demanda por gasolina no Brasil, no período de agosto de 1994 a julho de 2006, com base no modelo de correção de erros (MCE). Os resultados indicaram que a demanda por gasolina é inelástica no curto e longo prazo. $\mathrm{O}$ autor concluiu que a demanda por gasolina no país era inelástica em relação ao preço e à renda. Estimou-se também a função de demanda com uma variável binária para identificar os impactos da entrada dos veículos flex-fuel a partir de março de 2003. Esta variável apresentou um coeficiente de $-0,137$, sugerindo que, a partir desse período, a demanda por gasolina se tornou menos inelástica, passando de -0,197 para -0,334. Concluiu-se que o álcool hidratado foi se tornando um substituto menos imperfeito da gasolina $\mathrm{C}$, embora sua demanda tenha permanecido inelástica no mercado nacional.

Schünemann (2007) avaliou o impacto na demanda de gasolina automotiva no Brasil com a introdução dos veículos flex-fluel e a expansão do consumo do gás natural veicular (GNV) no passado recente. Os resultados sugeriram que a gasolina $\mathrm{C}$ é um bem inelástico em relação ao preço e à renda no curto prazo. No longo prazo, se torna um bem relativamente mais elástico à renda, porém, a inelasticidade em relação ao preço se mantém. Apesar de o preço do GNV ser relativamente mais 
competitivo que os preços do álcool e da gasolina, existe um elevado custo de conversão dos veículos para sua utilização. Assim, a opção pelo GNV é de ordem estrutural/tecnológica, pois o preço deste combustível não foi relevante em suas estimativas.

Vilela e Júnior (2010) analisaram a demanda por gasolina C nos Estados do Ceará, Pernambuco, Sergipe, Rio de Janeiro, Paraná, Rio Grande do Sul e Mato Grosso do Sul, de julho de 2001 a dezembro de 2012. Os autores avaliaram as diferentes reações da demanda às variações no preço desse combustível, mais especificamente via mudanças no imposto Estadual ICMS sobre a gasolina C, utilizando-se de técnicas de cointegração. Os resultados sugeriram que a demanda por gasolina C era inelástica em relação ao preço para os Estados em análise e para o Brasil. Entretanto, alguns coeficientes se revelaram estatisticamente não significativos. Os autores concluíram que as estratégias baseadas no uso de políticas públicas nacionais, que não considerassem as especificidades de cada Estado, provavelmente não seriam eficazes ou teriam menor eficácia.

Souza (2010) avaliou como as mudanças mais recentes impactaram sobre a demanda de combustível nos Estados brasileiros no período de 2001 a 2009. Para tanto, foram estimadas as elasticidades renda e preço (própria e cruzada) do consumo dos principais combustíveis para veículos leves. Os resultados confirmam a existência de um aumento das elasticidadespreço direta e cruzada do consumo de gasolina e do álcool hidratado no Brasil. Adicionalmente, as estimações sugeriram que, no período mais recente, o álcool apresentou em módulo uma elasticidade-preço significativamente maior que a gasolina.

\section{Metodologia}

O referencial teórico utilizado neste artigo refere-se à teoria microeconômica da demanda. Conforme Varian (2006), a demanda por um bem é função do seu preço, da renda, do preço do produto substituto, 
do preço do bem complementar e de outras variáveis, como gostos e preferências.

As variáveis que podem explicar a demanda por gasolina $\mathrm{C}$ são: preço desse combustível, preço de um bem substituto, renda do consumidor, frotas de veículos a gasolina e flex. Nesse estudo, a função de demanda por gasolina $\mathrm{C}$ apresentou, como variáveis explicativas, o preço da gasolina, o preço de um combustível substituto (álcool hidratado) e a renda do consumidor. Tais variáveis foram escolhidas, uma vez que existem na literatura evidências empíricas de que elas são relevantes para explicar a demanda de gasolina $C$ no país. As variáveis frota de veículos a gasolina e flex não foram incluídas no modelo, pois, no caso da primeira, existe uma elevada correlação entre ela e a renda do consumidor, o que gera resultados inconsistentes com a teoria econômica ${ }^{5}$. A respeito dos dados dos veículos flex, não se encontram disponíveis para o período em análise, uma vez que tais veículos foram lançados comercialmente no Brasil a partir de março de 2003. Portanto, as elasticidades estimadas neste trabalho podem estar subestimadas em razão da exclusão dessas variáveis na equação de demanda .

A função de demanda de gasolina C no Estado de Minas Gerais, estimada nesse trabalho, é representada por uma função do tipo Cobb-Douglas. Uma das principais propriedades desse tipo de função é o fato de as elasticidades serem constantes e iguais aos coeficientes das variáveis na forma logarítmica. Diante disso, esta função foi especificada da seguinte forma:

$$
Q g_{t}=C \cdot P g_{t}^{\beta_{1}} \cdot P a_{t}^{\beta_{2}} \cdot R_{t}^{\beta_{3}} \cdot e_{t}^{\varepsilon_{I}}
$$

em que $\mathrm{Qg}_{t}$ é quantidade consumida de gasolina $\mathrm{C}$ em metros cúbico; $\mathrm{Pg}_{\mathrm{t}}$ é o preço da gasolina $\mathrm{C}$, em reais, no varejo; $\mathrm{Pa}_{\mathrm{t}}$ é o preço do álcool hidratado, em reais, no varejo; $R t$, renda do consumidor (folha

5 Foi estimada uma equação de demanda com a inclusão dessa variável, porém, o vetor de cointegração gerado apresentou coeficientes com sinais inconsistentes em relação ao esperado. Tal fato talvez possa ser explicado pela elevada colinearidade existente entre a renda e as vendas de veículos movidos a gasolina.

6 Para mais informações a respeito da influência dessas variáveis sobre as elasticidades da demanda de gasolina, ver Costa e Guilhoto (2010). 
de pagamento real por trabalhador relativa à indústria geral em Minas Gerais) ; e termo de erro com pressuposições usuais; e $t$, tempo, periodicidade mensal.

A equação (1) pode ser tomada na forma de logaritmo natural e apresentada de forma linear, conforme a equação (2):

$\ln Q g_{t}=\ln C+\beta_{1} \ln P g_{t}+\beta_{2} \ln P a_{t}+\beta_{3} \ln R_{t}+\varepsilon_{I t}$

Espera-se que a demanda de gasolina $\mathrm{C}$ responda positivamente ao preço do álcool hidratado e à renda do consumidor $\left(\beta_{2}, \beta_{3}>0\right) \mathrm{e}$ negativamente ao preço da gasolina $\mathrm{C}\left(\beta_{1}<0\right)$. Os parâmetros $\beta_{i}{ }^{\prime}$ 's podem ser interpretados como as elasticidades da demanda de gasolina $\mathrm{C}$ em relação às variáveis explicativas supracitadas.

Existem pelo menos duas metodologias possíveis para se estimar uma função de demanda: pelo método de equações simultâneas ou por meio de cointegração. Para estimar a equação (2), optou-se por utilizar os procedimentos propostos por Johansen (1988) e Johansen e Juselius (1990). A metodologia consiste em estimar o(s) vetor(es) de cointegração a partir da modelagem de um vetor Autorregressivo (VAR) na primeira etapa do processo e, na sequência, estimar um Mecanismo de Correção de Erros (MCE) com vetor de cointegração. A opção por esses modelos se justifica pelo fato de as séries temporais analisadas serem não estacionárias. O conceito de cointegração, introduzido por Engle e Granger (1987), permite que as regressões que envolvem séries não estacionárias sejam realizadas sobre seus níveis, sem que se incorra no problema da regressão espúria. Além disso, as informações de longo prazo não são perdidas, o que ocorre quando são utilizadas séries diferenciadas.

A metodologia de Autorregressão Vetorial (VAR) foi proposta como alternativa aos modelos estruturais multiequacionais. Um VAR tem duas dimensões: número de variáveis $=k$ e número de defasagens $=p . \mathrm{Um}$ $\operatorname{VAR}(p)$ com $k$ variáveis é representado, em forma matricial, conforme Hamilton (1994), por: 


$$
Y_{t}=\alpha+\theta_{1} Y_{t-1}+\theta_{2} Y_{t-2}+\ldots \ldots+\theta_{p} Y_{t-p}+\varepsilon_{t}
$$

em que $Y_{t}$ é um vetor $k \times 1$ de variáveis endógenas; $Y_{t-j}$, $j=1,2,3, \ldots \ldots . ., p$ são vetores $k \times 1$ de variáveis defasadas; $\alpha$ é um vetor $k \times 1$ de interceptos; $\theta_{i}, i=1,2,3, \ldots \ldots . ., p$ são matrizes $k \times k$ de coeficientes a serem estimados; e $\varepsilon_{t}$ é um vetor $k \times 1$ de erros aleatórios $\operatorname{com} E\left(\varepsilon_{t}\right)=0$ e $E\left(\varepsilon_{t} \varepsilon_{t}^{\prime}\right)=\Omega$.

No entanto, o modelo requer a utilização de séries estacionárias em nível, caso contrário elas devem se tornar estacionárias por meio de uma transformação adequada dos dados. Para testar a estacionariedade das séries, utilizou-se o teste de Dickey e Fuller Aumentado (ADF), proposto por Dickey e Fuller (1981). Se a ordem de integração das séries temporais for a mesma, prossegue-se com os testes, fazendo-se a análise da existência de cointegração entre as variáveis. O conceito de cointegração está relacionado a uma relação de equilíbrio, no longo prazo, entre as variáveis.

Se houver a possibilidade de existir mais de um vetor de cointegração ou se houver endogeneidade nos regressores, o teste para a presença de cointegração é feito a partir de uma estrutura multivariada, sendo o modelo proposto por Johansen (1988 e 1991) o mais indicado. O procedimento de Johansen (1988 e 1991) baseia-se na versão reparametrizada de um modelo de Vetor Autorregressivo (VAR), com $p$ defasagens. O VAR restrito ou reparametrizado pode ser denominado de Vetor de Correção de Erro (VEC), sendo aplicado se as séries forem estacionárias por meio da diferenciação. O modelo VEC pode ser representado pela seguinte expressão:

$$
\Delta Y_{t}=\Gamma_{1} \Delta Y_{t-1}+\Gamma_{2} \Delta Y_{t-2}+\ldots \ldots+\Gamma_{p-1} \Delta Y_{t-(p-1)}+\Pi Y_{t-1}+\mu+\varepsilon_{t}
$$


em que $Y_{t}$ é um vetor com $\mathrm{k}$ variáveis; $\varepsilon_{t} \sim \mathrm{N}(0, \Sigma)$ e $\mathrm{E}\left(\varepsilon_{t}, \varepsilon^{\prime}{ }_{s}\right) \equiv 0$, $\operatorname{com} \mathrm{t} \neq \mathrm{s}$.

A partir do vetor de cointegração estimado, escreve-se a relação de equilíbrio no longo prazo da demanda de gasolina $\mathrm{C}$ no Estado de Minas Gerais e interpreta-se cada um dos coeficientes estimados como a elasticidade de longo prazo em relação a cada variável explicativa. A modelagem (VAR-VEC) é interessante por levar em consideração as variáveis e suas defasagens, conciliando as tendências de curto e longo prazos. Além disso, este enfoque é flexível o suficiente para se obter uma representação estatística dos dados sem a necessidade de introduzir variáveis ad-hoc a priori, como no caso dos modelos de equações simultâneas.

\subsection{Fonte de dados}

Os dados utilizados nesse trabalho foram mensais, de janeiro de 2002 a dezembro de 2010. As vendas pelas distribuidoras (em metros cúbicos) e os preços (em reais) da gasolina $\mathrm{C}$ e do álcool hidratado no mercado varejista do Estado de Minas Gerais foram coletados na Agência Nacional de Petróleo (ANP). A série que representou a renda média do consumidor mineiro foi a folha de pagamento real por trabalhador relativa à indústria geral em Belo Horizonte, obtida no junto ao Instituto Brasileiro de Geografia e Estatística (IBGE). Os preços dos combustíveis, gasolina C e álcool hidratado foram deflacionados pelo Índice Geral de Preço de Disponibilidade Interna (IGP-DI), obtido no site da Fundação Getúlio Vargas (FGV).

\section{Resultados e discussões}

A estimação da demanda de gasolina C, a partir do modelo de Autorregressão Vetorial (VAR) reparametrizado requer a realização 
prévia de testes estatísticos (raiz unitária e cointegração) para assegurar a validação de sua utilização. Inicialmente, foi feito o Teste de Raiz Unitária de Dickey-Fuller (DF) Aumentado (ADF) para testar a estacionariedade das séries estudadas. Os resultados obtidos encontram-se reportados na Tabela 1:

Tabela 1 - Teste de Raiz Unitária ADF das séries $\operatorname{lnQg}, \ln P g, \ln P a, \ln R$ no período de janeiro de 2002 a dezembro de 2010

\begin{tabular}{cccc}
\hline Série & Defasagem & Integração & Resultado do teste ADF \\
\hline $\operatorname{LnQg}$ & 1 & $\mathrm{I}(1)$ & $-2,634^{*}$ \\
\hline $\mathrm{LnPg}$ & 0 & $\mathrm{I}(1)$ & $-8,450^{* * *}$ \\
\hline $\mathrm{LnPa}$ & 0 & $\mathrm{I}(1)$ & $-12,259^{* * *}$ \\
\hline $\mathrm{LnR}$ & 1 & $\mathrm{I}(1)$ & $-6,309^{* * *}$ \\
\hline
\end{tabular}

Fonte: Resultados da pesquisa.

***Significativo a $1 \% \mathrm{e} *$ Significativo a $10 \%$.

Considerando as séries em nível, não se pode rejeitar a hipótese da existência de raiz unitária. Portanto, foi necessário utilizar a primeira diferença para torná-las estacionárias aos níveis de significância de 1\% e 10\%.

Como as séries foram integradas de mesma ordem I(1), o passo subsequente foi testar a existência de cointegração entre elas, utilizando o procedimento de Johansen a partir da especificação de um modelo VAR. Na identificação do número de defasagens do modelo VAR, foram utilizados os Critérios de Akaike e Schwarz (Tabela 2). 
Tabela 2 - Definição do número de defasagens do modelo VAR pelos Critérios de Informação de Akaike e Schwarz

\begin{tabular}{ccc}
\hline Defasagem & Akaike & Schwarz \\
\hline 0 & -12.70126 & -13.79734 \\
\hline 1 & $-12.92865^{*}$ & $-13.83468^{*}$ \\
\hline 2 & -12.91023 & -13.59233 \\
\hline 3 & -12.77271 & -13.27715 \\
\hline 4 & -12.61919 & -12.94323 \\
\hline
\end{tabular}

Nota:* Indica o número de defasagens escolhido por cada um dos critérios.

Fonte: Resultados da pesquisa.

De acordo com ambos os critérios de informação, o modelo VAR deve conter apenas uma defasagem. Na sequência, foram feitos os testes do Traço e do Máximo Autovalor, cujo objetivo foi encontrar os vetores de cointegração caso existisse relação de longo prazo entre as variáveis em análise. A Tabela 3 reporta os resultados dos testes de Traço e do Máximo Autovalor para cointegração entre as variáveis $\mathrm{LnQg}_{\mathrm{t}}, \mathrm{LnPg}_{\mathrm{t}}, \mathrm{LnPa}_{\mathrm{t}}, \mathrm{LnR}_{\mathrm{t}}$.

Tabela 3 - Teste do Traço e do Máximo-Autovalor para cointegração entre as variáveis $\mathrm{LnQg}_{\mathrm{t}}, \mathrm{LnPg}_{\mathrm{t}}, \mathrm{LnPa}_{\mathrm{t}}, \mathrm{LnR}_{\mathrm{t}}$

\begin{tabular}{cccccc}
\hline $\begin{array}{c}\text { Hipótese } \\
\left(\mathrm{H}_{0}\right)\end{array}$ & $\left(\mathrm{H}_{1}\right)$ & $\begin{array}{c}\text { Teste do Traço } \\
\text { Estatística do teste }\end{array}$ & $\begin{array}{c}\text { Hipótese } \\
\left(\mathrm{H}_{0}\right)\end{array}$ & $\begin{array}{c}\text { Teste do Máximo } \\
\left(\mathrm{H}_{1}\right)\end{array}$ & $\begin{array}{c}\text { Autovalor } \\
\text { Estatística do teste }\end{array}$ \\
\hline $\mathrm{r}=0$ & $r \geq 1$ & $63,945^{* * *}$ & $\mathrm{r}=0$ & $\mathrm{r}=1$ & $35,346^{* * *}$ \\
\hline $\mathrm{r} \leq 1$ & $r \geq 2$ & 28,598 & $r \leq 1$ & $\mathrm{r}=2$ & 16,259 \\
$\mathrm{r} \leq 2$ & $r \geq 3$ & 12,339 & $r \leq 2$ & $\mathrm{r}=3$ & 7,941 \\
\hline $\mathrm{r} \leq 3$ & $r \geq 4$ & 4,398 & $r \leq 3$ & $\mathrm{r}=4$ & 4,398 \\
\hline
\end{tabular}

Nota:***Significativo ao nível de $1 \%$.

Fonte: Resultados da pesquisa.

Na Tabela 3, verifica-se que, segundo o teste de traço, a hipótese de que o posto da matriz de cointegração seja nulo é rejeitada, entretanto não se rejeita a hipótese $r \geq 1$, ao nível de significância de $1 \%$. Da mesma forma, de acordo com o teste do Máximo Autovalor, a hipótese de que o posto da matriz de cointegração seja nulo é rejeitada ao nível de significância de $1 \%$. A este nível de significância, não se rejeita a hipótese alternativa 
$r=1$. Portanto, pode-se concluir que existe uma relação de equilíbrio no longo prazo entre as variáveis (Tabela 4).

Tabela 4 - Vetor de cointegração normalizado pelo método de Johansen, janeiro de 2002 a dezembro de 2010

\begin{tabular}{lllll}
$\mathrm{LnQg}_{\mathrm{t}}$ & Constante & $\mathrm{LnPg}_{\mathrm{t}}$ & $\mathrm{LnPa}_{\mathrm{t}}$ & $\mathrm{LnR}_{\mathrm{t}}$ \\
\hline 1,0000 & $-0,7918^{* * *}$ & $0,0583^{* * *}$ & $-0,1386^{* * *}$ & $-1,0001^{* * *}$ \\
& $(0,58397)$ & $(0,37870)$ & $(0,20136)$ & $(0,12875)$ \\
\hline
\end{tabular}

Notas: As estatísticas entre parênteses referem-se ao desvio-padrão do parâmetro estimado.

$(* * *)$ significativo a $1 \%$.

Fonte: Resultados da pesquisa.

O resultado do vetor de cointegração estimado, conforme a Tabela 4, representa a relação de longo prazo da demanda de gasolina $\mathrm{C}$ no mercado varejista do Estado de Minas Gerais e os seus principais determinantes. Além disso, como todas as variáveis foram tomadas em logaritmo, os coeficientes estimados representam as elasticidades de longo prazo da demanda de gasolina $\mathrm{C}$ em relação a cada uma das variáveis explicativas. Tal relação pode ser expressa pela equação (5):

$$
\operatorname{LnQg} g_{t}=0,7918-0,0583 \operatorname{LnPg}_{t}+0,1386 \operatorname{LnPa} a_{t}+1,0001 \operatorname{LnR} R_{t}
$$

Todos os parâmetros estimados apresentaram sinais coerentes com o sugerido pela teoria econômica e foram estatisticamente significativos.

A elasticidade-preço direta da demanda, medida pelo coeficiente do logaritmo do preço da gasolina $\mathrm{C}$, sugeriu que, tudo mais permanecendo constante, um acréscimo (decréscimo) de $10 \%$ no preço da gasolina $\mathrm{C}$ no mercado varejista induz uma redução (aumento) de $0,583 \%$ na quantidade demandada de gasolina C. Portanto, a demanda por gasolina C no Estado de Minas Gerais é inelástica em relação ao preço no longo prazo. Esse resultado é coerente com aqueles encontrados nos trabalhos de Alves e Bueno (2003), Roppa (2005), Nappo (2007), Schunemann (2007) e Souza (2010) para o mercado brasileiro (Tabela 5). 
Tabela 5 - Resultados das estimativas da elasticidade preço da demanda por gasolina para o Brasil

\begin{tabular}{lcc}
\hline Estudo & Periodicidade & Elasticidades de longo prazo \\
\hline Alves e Bueno (2003) & $1974-1999$ (anual) & $-0,465$ \\
Roppa (2005) & $1979-2000$ (anual) & $-0,634$ \\
Nappo (2007) & $1994-2006$ (mensal) & $-0,197$ \\
Schunemann (2007) & $2001-2007$ (mensal) & $-0,22$ \\
Souza (2010) & $2001-2009$ (mensal) & $-0,37$ \\
Presente Estudo (MG) & $2002-2010$ (mensal) & $-0,058$ \\
\hline
\end{tabular}

Fonte: Elaboração própria.

A Tabela 5 apresenta as elasticidades estimadas para o Brasil em diferentes periodicidades e o resultado deste trabalho para Minas Gerais. Assim como o presente estudo, os trabalhos supracitados utilizaram o modelo de correção de erros para calcular as relações de longo prazo quando as variáveis são cointegradas. Verifica-se, portanto, que existem evidências empíricas na literatura de que a demanda é inelástica também no mercado nacional.

Com relação à elasticidade-preço cruzada, verificou-se que, tudo o mais permanecendo constante, um aumento (diminuição) de $10 \%$ no preço do álcool hidratado implica um aumento (redução) de 1,386\% na quantidade demandada de gasolina C. A correlação positiva entre o preço do álcool hidratado e a quantidade demandada de gasolina $\mathrm{C}$ sugere que ambos os combustíveis são substitutos entre si no Estado de Minas Gerais, embora o grau de substitubilidade entre eles seja imperfeito mesmo no longo prazo. Conforme ressaltou Borba (2008), além do preço de mercado, diversos fatores podem influenciar os consumidores no momento de decisão quanto ao combustível a utilizar para abastecer o seu veículo, diante da possibilidade de se fazer tal escolha, como, por exemplo, a confiança na tecnologia flex e os fatores sociais. Deve-se lembrar também que a gasolina permite percorrer uma distância maior do que o álcool, reduzindo o número de paradas para abastecimento. Tudo isso pode explicar pelo menos em parte, os resultados obtidos para a magnitude da elasticidade-preço cruzada da demanda. 
Sobre a elasticidade renda da demanda de gasolina C no Estado de Minas Gerais, verifica-se que, no longo prazo, um aumento (decréscimo) de $10 \%$ na renda dos consumidores mineiros poderá elevar (diminuir) a demanda por gasolina C em 10,001 \%, ou seja, os acréscimos ocorrem praticamente na mesma proporção.

A análise de cointegração permite ainda obter os coeficientes de ajustamento de curto prazo, que refletem a velocidade de ajustamento de curto prazo das variáveis em direção ao equilíbrio de longo prazo. Mediante um desequilíbrio transitório, de curto prazo, um elevado coeficiente indica que a velocidade de ajustamento em direção ao longo prazo é elevada. Por outro lado, se os resultados apresentarem baixa magnitude desse coeficiente, a transição de um desequilíbrio de curto prazo para um equilíbrio no longo prazo tende a ser corrigida mais lentamente.

A Tabela 6 apresenta os resultados do ajustamento de curto prazo para cada uma das variáveis logartimizadas $\operatorname{LnQg}_{t}, \operatorname{LnPg}_{t}, \operatorname{LnPa}_{t}, \operatorname{LnR}_{t}$

Tabela 6 - Coeficientes de ajustamento do Modelo de Correção de Erros Irrestrito, janeiro de 2002 a dezembro de 2010.

\begin{tabular}{llll}
\hline LnQgt & LnPgt & LnPat & LnRt \\
\hline $0,0667^{* * *}$ & $-0,0417^{* * *}$ & $0,1432 * * *$ & $0,6569 * * *$ \\
$(0,0814)$ & $(0,022)$ & $(0,0832)$ & $(0,1160)$ \\
\hline
\end{tabular}

Notas: As estatísticas entre parênteses referem-se ao desvio-padrão do parâmetro estimado.

$(* * *)$ significativo a $1 \%$.

Fonte: Dados da pesquisa.

No curto prazo, o coeficiente do logaritmo da quantidade demandada de gasolina $\mathrm{C}$ no mercado varejista mineiro $\left(\mathrm{LnQg}_{\mathrm{t}}\right)$ foi de 0,0667 , evidenciando que os desequilíbrios transitórios para essa variável são corrigidos a uma velocidade de $6,67 \%$, o que representa uma baixa velocidade de ajustamento.Assim como nos resultados de longo prazo, 
todos os coeficientes foram estatisticamente significativos e apresentaram sinais coerentes com a teoria econômica. Os coeficientes da elasticidadepreço da demanda apresentaram comportamentos economicamente consistentes, uma vez que se revelaram maiores no longo prazo quando comparados aos de curto prazo. Entretanto, em ambos os períodos, o consumidor mineiro é inelástico em relação às variações no preço da gasolina C. No longo prazo, os consumidores terão tempo o suficiente para buscar alternativas de locomoção e abastecimento, quando ocorrerem elevações no preço da gasolina C. O coeficiente do logaritmo do preço do álcool hidratado se revelou positivamente correlacionado com a quantidade demandada de gasolina $\mathrm{C}$, evidenciando que, no curto prazo, ambos os combustíveis são substitutos entre si. O baixo valor encontrado para este coeficiente pode refletir o grau de imperfeição da substitubilidade entre ambos. Sobre a elasticidade renda da demanda no curto prazo, verificou-se que um aumento (diminuição) de $10 \%$ na renda do consumidor mineiro implica um aumento (redução) de 6,57\% na quantidade demandada de gasolina $\mathrm{C}$, evidenciando que, no longo prazo, o consumidor mineiro torna-se mais sensível às variações na renda quando comparado ao curto prazo.

\section{Conclusão}

Este trabalho teve como objetivo analisar a demanda de gasolina $\mathrm{C}$ no Estado de Minas Gerais, no período de janeiro de 2002 a dezembro de 2010. Para tal, estimou-se um modelo de Autorregressão Vetorial (VAR) reparametrizado, denominado Vetor de Correção de Erro (VEC).

Os resultados sugeriram que a demanda de gasolina $\mathrm{C}$ no Estado de Minas Gerais é influenciada pelo preço da gasolina C, preço do álcool hidratado e renda do consumidor mineiro. Além disso, verificou-se que ela é inelástica em relação a seu preço, no curto e no longo prazos, entretanto, o coeficiente da elasticidade-preço da demanda foi comparativamente superior no longo prazo. Esse resultado já era esperado, uma vez que, no longo prazo, o consumidor tem mais tempo para buscar alternativas de locomoção e abastecimento. A elasticidade-preço cruzada definiu o álcool 
hidratado como um substituto imperfeito para a gasolina $\mathrm{C}$ em ambos os períodos. A respeito das variações na renda, verificou-se que a demanda foi inelástica no curto prazo, de modo que um aumento (diminuição) de $10 \%$ na renda do consumidor mineiro implica um aumento (redução) de $6,57 \%$ na quantidade demandada de gasolina C. Por outro lado, no longo prazo, um aumento (decréscimo) de 10\% na renda dos consumidores mineiros, poderá elevar (diminuir) a demanda por gasolina C em 10,001 $\%$, ou seja, as variações ocorrem praticamente na mesma proporção.

Em síntese, é importante ressaltar que as variáveis analisadas nesse trabalho (preço da gasolina $\mathrm{C}$, preço do álcool hidratado e renda) foram relevantes para explicar a variabilidade na quantidade demandada de gasolina C em Minas Gerais, no período de janeiro de 2002 a dezembro de 2010. Conforme salientado anteriormente, existem evidências empíricas na literatura de que tais variáveis são importantes para explicar a demanda de gasolina comum também no mercado nacional. Entretanto, outros elementos também podem ser relevantes para explicar a demanda de gasolina C nesse Estado. Diante disso, para trabalhos futuros, recomenda-se a realização de análises que procurem identificar outras variáveis determinantes da demanda de gasolina C neste Estado, como, por exemplo, o preço do gás natural veicular, custo de utilização de transportes públicos, as vendas de automóveis e comerciais leves e/ ou a frota de veículos, entre outros. A respeito da frota de veículos flex, ressalta-se que os dados se encontram disponíveis somente a partir de março de 2003.

\section{Referências}

AGÊNCIA NACIONAL DE PETRÓLEO - ANP. Vendas pelas distribuidoras dos derivados de combustíveis de petróleo (metros cúbicos). Disponível em <http://www.anp.gov.br/ doc/dados_estatisticos/Vendas_de_Combustiveis_m3.xls. Acesso em fev. 2012. 
ALVES, D. C. O.; BUENO, R. L. S. Short-run, long-run and cross elasticities of gasoline demand in Brazil. Energy Economics. EUA: v. 25, p. 191-199. Março de 2003.

BACCHI, M. R. P. (2005). Formação de preços no setor sucroalcooleiro da região centro-sul do Brasil: relação com o mercado de combustível fóssil. In: XXXIII Encontro Nacional de Economia. Associação Nacional dos Centros de Pós-Graduação em Economia, Natal - RN.

BORBA, B. S. M. C.. Metodologia de regionalização do mercado de combustíveis automotivos no Brasil. 136 p. Dissertação (Mestrado Planejamento Energético) - Universidade Federal do Rio de Janeiro, Rio de Janeiro, 2008.

BURNQUIST, H.L., BACCHI, M.R.P. A demanda por gasolina no Brasil: uma análise utilizando técnicas de cointegração. CEPEA. 2001. Disponível em: <www.cepea.esalq.usp.br/pdf/DemandaGasolina. pdf>. Acessoem: 15/01/2012.

DICKEY, D. A.; FULLER, W. A. Distribution of estimates for autoregressive time series with unit root. Journal of American Statistics Association, v. 74, n. 366, p. 427-431, 1979.

ENGLE, R.F.; GRANGER, C.W. Co-integration and error-correction: representation, estimation and testing. Econometrica, v. 55, n. 1, p. 251-276, 1987.

FUNDAÇÃO GETÚlio VARGAS. Índice Geral de Preços Disponibilidade interna (Índice 2). Disponível em: $<$ http:// www. indicadores.hpg.ig.com.br>. Acesso em: 20 jun. 2012.

COSTA, C. C.; GUILHOTO, J. J. M. Impacto da diferenciação do ICMS entre etanol hidratado e gasolina $\mathrm{C}$ para a economia do estado de Minas Gerais. In: VIII Encontro Nacional Enaber, 2010, Juiz de Fora, MG. Anais... VIII Encontro Nacional Enaber, 2010. 
HAMILTON, J.D. Time series analysis. Princeton, New Jersey: Princeton University, 1994.

INSTITUTO BRASILEIRO DE GEOGRAFIA E ESTATÍSTICA(IBGE). Disponível em:<http://www.ibge.gov.br/>. Acesso em: 12 nov. 2011.

JOHANSEN, S., Statistical Analysis of Cointegration Vectors. Journal of Economic Dynamics and Control v.12, n. , p. 231-254, 1988.

JOHANSEN, S., JUSELIUS, K. Maximum likelihood estimation and inference on cointegration - with application to the demand for money. Oxford Bulletin on Economics and Statistics, v. 52, n. 1, p. 169-210, 1990.

NAPPO, M..A demanda por gasolina no Brasil: Uma avaliação de suas elasticidades após a introdução dos carros biocombustível. 61p. Dissertação (Mestrado) - Escola de Economia de São Paulo, Fundação Getúlio Vargas - FGV, São Paulo, 2007.

ROPPA, B. F. (2005). Evolução do consumo de gasolina no Brasil e suas elasticidades: 1973 a 2003. Trabalho de Conclusão de Curso, Instituto de Economia da Universidade Federal do Rio de Janeiro, Rio de Janeiro, Brasil.

SCHÜNEMANN, L. (2007). A demanda de gasolina automotiva no Brasil: o impacto nas elasticidades de curto e longo prazo da expansão do GNV e dos carros flex. 107p. (Dissertação de Mestrado). Faculdades de Economia e Finanças do Ibmec. Rio de Janeiro: Faculdades Ibmec. 2007.

SILVÉRIO, R. P. Avaliação dos impactos dos veículos flex na elasticidade-preço da demanda por etanol no Estado do Rio de Janeiro entre 2001 e 2007. Rio de Janeiro: Universidade Federal do Rio de Janeiro, 2007. Monografia de Graduação - Curso em Ciências Econômicas do Instituto de Economia da Universidade Federal do Rio de Janeiro. 
SOUZA, A. N. Estudo das demandas de etanol e gasolina no Brasil no período 2001 - 2009. 60 p. Dissertação (mestrado profissional) Escola de Economia de São Paulo, Fundação Getúlio Vargas - FGV, São Paulo, 2010.

VARIAN, H.R. Microeconomia: princípios básicos. $7^{\circ}$ ed. Rio de Janeiro: Campus, 2006. 778 p.

VILELA, T. M.M, PINTO JÚNIOR, H. Q. Análise de sensibilidade do consumo de gasolina $\mathrm{C}$ entre julho de 2001 e dezembro de 2008: política tributária estadual como instrumento de políticas energéticas e ambientais. Revista Nova Economia, v. 20, n.3, p.403-426, 2010. 